\title{
Non-invasive Methods in Diagnosis of Nonalcoholic Fatty Liver Disease Khaled Hemida $^{1}$, Hany Haroun ${ }^{1}$, Mostafa Mahmoud ${ }^{2}$, Inas Elkhedr Mohamed*1 \\ ${ }^{1}$ Department of Gastroenterology, Faculty of Medicine, Ain Shams University, Egypt. \\ ${ }^{2}$ El Sahel Hospital, Ministry of Health, Egypt \\ *Corresponding author: Inas Elkhedr Mohamed, Mobile: (+20) 01093351447, E-Mail: inas_elkhedr@yahoo.com
}

\begin{abstract}
Background: Non-alcoholic fatty liver disease (NAFLD) is a risk factor for increased morbidity and mortality plus cardiovascular disease and malignancy. Despite all of these, most patients have good prognosis if diagnosed at early stages. Objective: The study was designed to evaluate different non-invasive methods as a diagnostic tool of nonalcoholic fatty liver disease among diabetic and non-diabetic patients. Patients and Methods: The study was done on 100 patients were divided into two groups of 50 diabetics and 50 non-diabetics with fatty liver disease on abdominal ultrasonography finding. These patients were assessed with transient elastography (TE) after lab tests and then scoring systems (Fib-4 and NAFLD fibrosis score) and liver stiffness scores were compared between the two groups. Results: There was statistically significant difference between the two groups as regard Fib-4 and NAFLD fibrosis score ( $p<0.001$ ), where Fib-4 and NAFLD fibrosis score were higher in group I (diabetic) than in group II (nondiabetic). A statistically significant difference between the two groups as regard liver stiffness $(\mathrm{p}<0.001)$, where liver stiffness was higher in group I than in group II. Liver stiffness score and NAFLD fibrosis score were statistically significantly higher in those with $(>\mathrm{F} 2)$ than those with (F2 or less) ( $\mathrm{p}<0.001)$. Conclusion: A combination of Fibroscan, Fib-4 and NAFLD fibrosis score provides a valuable non-invasive method in diagnosis of NAFLD patients, and this can eliminate the need for liver biopsy in patients without clear indication, especially diabetic patients that can help in early diagnosis of NAFLD before development of fibrosis.
\end{abstract}

Keywords: FIB-4, Fibroscan, NAFLD fibrosis score, Non-alcoholic fatty liver disease.

\section{INTRODUCTION}

Non alcoholic fatty liver disease (NAFLD) is one of the most common chronic liver diseases, it has strong correlation with metabolic syndrome, and cardiovascular risk factors ${ }^{(\mathbf{1})}$.

According to the pathological changes, NAFLD is classified into simple steatosis, NASH and associated cirrhosis ${ }^{(2,3)}$. Simple steatosis is the fatty histological change in the liver, NASH is the disorder of hepatic cell with inflammatory activity or damage that can lead to cirrhosis or hepatocellular carcinoma $(\mathbf{1 , 4 )}$. The first change of NAFLD is fatty degeneration of liver. In order to control the progress of all the severe hepatic diseases, effective diagnostic tools are necessary to determine the severity of steatosis for the assessment and treatment of NAFLD ${ }^{(5)}$.

Liver biopsy is the gold standard of the diagnosis of NAFLD, but it is invasive. Therefore, to find the effective non-invasive diagnostic approach of NAFLD is an important goal worldwide (6). Non-invasive diagnostic approaches are studied. Currently available tests rely on two different but complementary approaches: a 'biological' approach based on serum biomarkers of fibrosis and a 'physical imaging' approach based on the measurement of liver stiffness using transient elastography ${ }^{(7)}$.

This study was designed to evaluate different non-invasive methods as a diagnostic tool of nonalcoholic fatty liver disease among diabetic and nondiabetic patients.

\section{PATIENTS AND METHODS}

A cohort study conducted over a period between January 2017 and June 2017. 100 patients were selected from Hepatology and Gastroenterology clinics, Ain Shams University Hospitals and El- Sahel Teaching Hospital with fatty liver disease.

Inclusion criteria: The patients diagnosed as NAFLD; their age ranged from 20-70 years.

Patients were classified into two groups: Group I: 50 diabetic patients with non-alcoholic fatty liver disease. Group II: 50 non-diabetic patients with non-alcoholic fatty liver disease.

The following patients were excluded from the study: heavy alcohol consumption (>40 g pure alcohol per day), positive history of HBV/HCV infection or drug induced hepatitis, patients on hepatotoxic medications such as: tamoxifen, diltiazem, methotrexate, corticosteroids, amiodarone, estrogen and antiretrovirals drugs, morbid obesity (BMI $\geq 40$ $\mathrm{Kg} / \mathrm{m}^{2}$ ), presence of other liver comorbidity including haemochromatosis, Wilson's disease, alantitrypsin deficiency, autoimmune hepatitis and bilharziasis, and immune suppression.

\section{Ethical considerations}

This study was performed in accordance with the ethical standards. The Faculty's Ethical Committee approval of Ain Shams University was taken before starting the study. Written informed consent was 
obtained from all participants before enrolment in the study.

All participants were subjected to the following: full medical history, thorough clinical examination, anthropometric measurements: including weight $(\mathrm{Kg})$, height $(\mathrm{cm})$ to calculate body mass index (BMI).

\section{I- Laboratory investigation including:}

1-Liver function tests (AST, ALT, y-glutamyltransferase GGT, total bilirubin), kidney functions, CBC.

2-Blood test (haptoglobin, $\quad$ 2-macroglobulin, apolipoprotein A1).

3- Serological markers (to exclude Wilson's disease, $\alpha 1$-antitrypsin deficiency and bilharziasis).

4- Antinuclear antibody (ANA) test (to exclude autoimmun hepatitis).

5- Iron profile: Serum iron, ferritin and total iron binding capacity

6- Viral markers for hepatitis patter (antigen and antibody B).

\section{II- Scoring systems:}

1-NAFLD fibrosis score: It incorporates different variables including (age, BMI, fasting hyperglycemia or previously diagnosed diabetes mellitus, platelet count, albumin, and AST/ALT ratio). With equation $\left\{\left(-1.76+0.037 \mathrm{X}\right.\right.$ age $(\mathrm{yr})+0.094 \mathrm{X}$ BMI $\left(\mathrm{Kg} / \mathrm{m}^{2}\right)+$ $1.13 \mathrm{X}$ impaired fasting glucose/diabetes mellitus $($ yes $=1$, no $=0)+0.99 \mathrm{X}$ AST/ALT ratio $-0.013 \mathrm{X}$ platelet $(\mathrm{X} 109 / \mathrm{L})-0.66 \mathrm{x}$ albumin $(\mathrm{g} / \mathrm{L})\}^{\left({ }^{(8)}\right.}$.

2-FIB-4 (fibrosis-4) index: It incorporates different variables including (age, AST, ALT and platelet), with equation ${ }^{(9)}$.

$$
\text { FIB-4= } \frac{\operatorname{Age}(y) X \operatorname{AST}(U / L)}{\text { Plateletcount } 10^{9} / L X \sqrt{\operatorname{ALT}(U / L)}}
$$

III- Abdominal ultrasonography: with the following characteristics (1) attenuation of image quickly within 4-5 $\mathrm{cm}$ of depth, making deeper structures difficult to decipher; (2) echogenic diffusely but particularly important to note brightness within the first $2-3 \mathrm{~cm}$ of depth; (3) liver uniformly heterogeneous; (4) thick subcutaneous depth (> $2 \mathrm{~cm})$; and (5) liver fills entire field with no edges visible ${ }^{(10)}$.

\section{IV-Fibroscan:}

All participants underwent transient elastography, using both ultrasounds of $5 \mathrm{MHz}$ and low frequency elastic waves. The system consists of a probe with an ultrasonic transducer mounted on the axis of a vibrator $(\mathbf{1 1 , 1 2 )}$. Measurements were performed on the right lobe of the liver through intercostals spaces between 25 and $45 \mathrm{~mm}$ from the skin surface. For each patient, the obtained elasticity value is the median of several measurements (usually 10) and the results are expressed in kilopascals $(\mathrm{kPa})$, results less than $13 \mathrm{kpa}$ race non cirrhotics (f0- f1- f2- f3), but in cirrhotic patients liver stiffness ranges from $13-15 \mathrm{kpa}$ to 75 $\mathrm{kpa}^{(13,14)}$

\section{Statistical analysis}

Data were analyzed using Statistical Package for the Social Sciences (SPSS) version 20.0. Quantitative data were expressed as mean \pm standard deviation (SD). Qualitative data were expressed as frequency and percentage. Independent-samples t-test of significance was used when comparing between two means. Spearman correlation was used to illustrate correlation between different scores. P-value $<0.05$ was considered significant. P-value $<0.001$ was considered as highly significant. $\mathrm{P}$-value $>0.05$ was considered insignificant.

\section{RESULTS}

The study included 100 patients with non alcoholic fatty liver disease 50 diabetic patients 29 of them were males (58\%) and 21 females (42\%). 50 non diabetics 25 $(50 \%)$ were males, and $25(50 \%)$ were females. The age of male patients ranged from (38-64 years) and ranged from (40-62 years) among females. Fib-4 and NAFLD fibrosis score were statistically significantly higher in diabetic group than in non-diabetic group (Table 1).

Table (1): Scoring systems distribution between diabetic and non-diabetic groups

\begin{tabular}{|l|c|c|c|c|c|}
\hline \multirow{2}{*}{} & \multicolumn{2}{|c|}{ Diabetic } & \multicolumn{2}{c|}{ Non Diabetic } & \multirow{2}{*}{ P value } \\
\cline { 2 - 5 } & Mean & $\begin{array}{c}\text { Standard } \\
\text { Deviation }\end{array}$ & Mean & $\begin{array}{c}\text { Standard } \\
\text { Deviation }\end{array}$ & \\
\hline NAFLD fibrosis score & -0.49 & 1.34 & -1.31 & 1.41 & $<0.001^{*}$ \\
\hline Fib_4 score & 2.22 & 0.99 & 1.52 & 0.70 & $<0.001^{*}$ \\
\hline
\end{tabular}

Liver stiffness was statistically significantly higher in diabetic group than in non-diabetic group (Table 2).

Table (2): Liver stiffness distribution between diabetic and non-diabetic groups

\begin{tabular}{|l|c|c|c|c|c|}
\hline & \multicolumn{2}{|c|}{ Diabetic } & \multicolumn{2}{c|}{ Non Diabetic } & \multirow{2}{*}{ P value } \\
\cline { 2 - 5 } & Mean & $\begin{array}{c}\text { Standard } \\
\text { Deviation }\end{array}$ & Mean & $\begin{array}{c}\text { Standard } \\
\text { Deviation }\end{array}$ & \\
\hline Liver stiffness $(\mathrm{K} \mathrm{Pa})$ & 8.67 & 3.28 & 6.37 & 2.08 & $<0.001^{*}$ \\
\hline
\end{tabular}

There was significant positive correlation between liver stiffness score and (BMI, Age, fasting blood glucose (F.B.G), $2 \mathrm{~h}$ postprandial blood glucose and TG), while there was significant negative correlation between liver stiffness score and (ALT and AST) (Table 3). 
https://ejhm.journals.ekb.eg/

Table (3): Correlation between Liver stiffness scores and other parameters

\begin{tabular}{|c|c|c|}
\hline Variables & \multicolumn{2}{|c|}{ Correlation } \\
\hline & $\mathbf{R}$ & P value \\
\hline BMI & $0.735^{* *}$ & $<0.001^{*}$ \\
\hline Age & $0.282^{* *}$ & $0.005^{*}$ \\
\hline ALT & -0.086 & 0.396 \\
\hline AST & -0.013 & 0.894 \\
\hline F.B.G & $0.660^{* *}$ & $<0.001^{*}$ \\
\hline 2h postprandial & $0.515^{* *}$ & $<0.001^{*}$ \\
\hline TG & 0.035 & $0.001^{*}$ \\
\hline
\end{tabular}

Liver stiffness score and NAFLD fibrosis score were statistically significantly higher in those with $(>\mathrm{F} 2)$ than those with (F2 or less)

(Table 4 and figures 1 and 2).

Table (4): Differences in liver stiffness score, Fib-4 score and NAFLD fibrosis score between patients with mild to moderate fibrosis and those with advanced fibrosis

\begin{tabular}{|l|l|l|l|l|l|}
\hline \multirow{2}{*}{} & \multicolumn{3}{|c|}{ Fibrosis stages } & \multirow{2}{*}{ P value } \\
\cline { 2 - 5 } & \multicolumn{2}{|c|}{ F2 or less } & \multicolumn{2}{|c|}{$>$ F2 (F3 or F4) } \\
\cline { 2 - 5 } & Mean & $\begin{array}{l}\text { Standard } \\
\text { Deviation }\end{array}$ & Mean & $\begin{array}{c}\text { Standard } \\
\text { Deviation }\end{array}$ & \multirow{2}{*}{$<0.001^{*}$} \\
\hline Fib_4 & 1.30 & 0.45 & 2.96 & 0.51 & $<0.001^{*}$ \\
\hline Liver stiffness (K Pa) & 5.81 & 1.50 & 10.84 & 2.18 & $<0.001^{*}$ \\
\hline NAFLD fibrosis score & -1.69 & 1.12 & 0.63 & 0.14 & \\
\hline
\end{tabular}

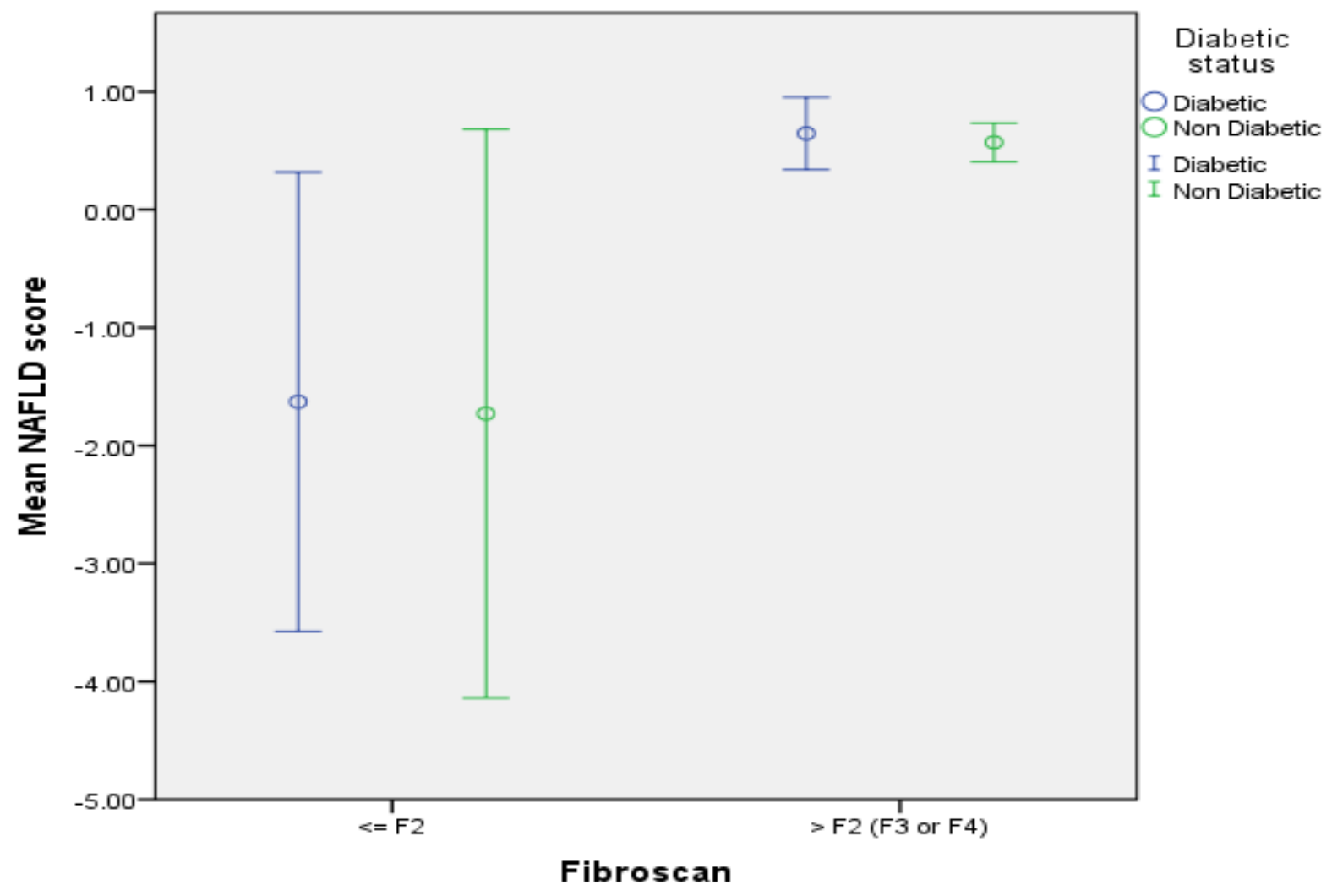

Error Bars: +/- $2 \mathrm{SD}$

Figure (1): Difference between NAFLD fibrosis score and liver fibrosis stages 


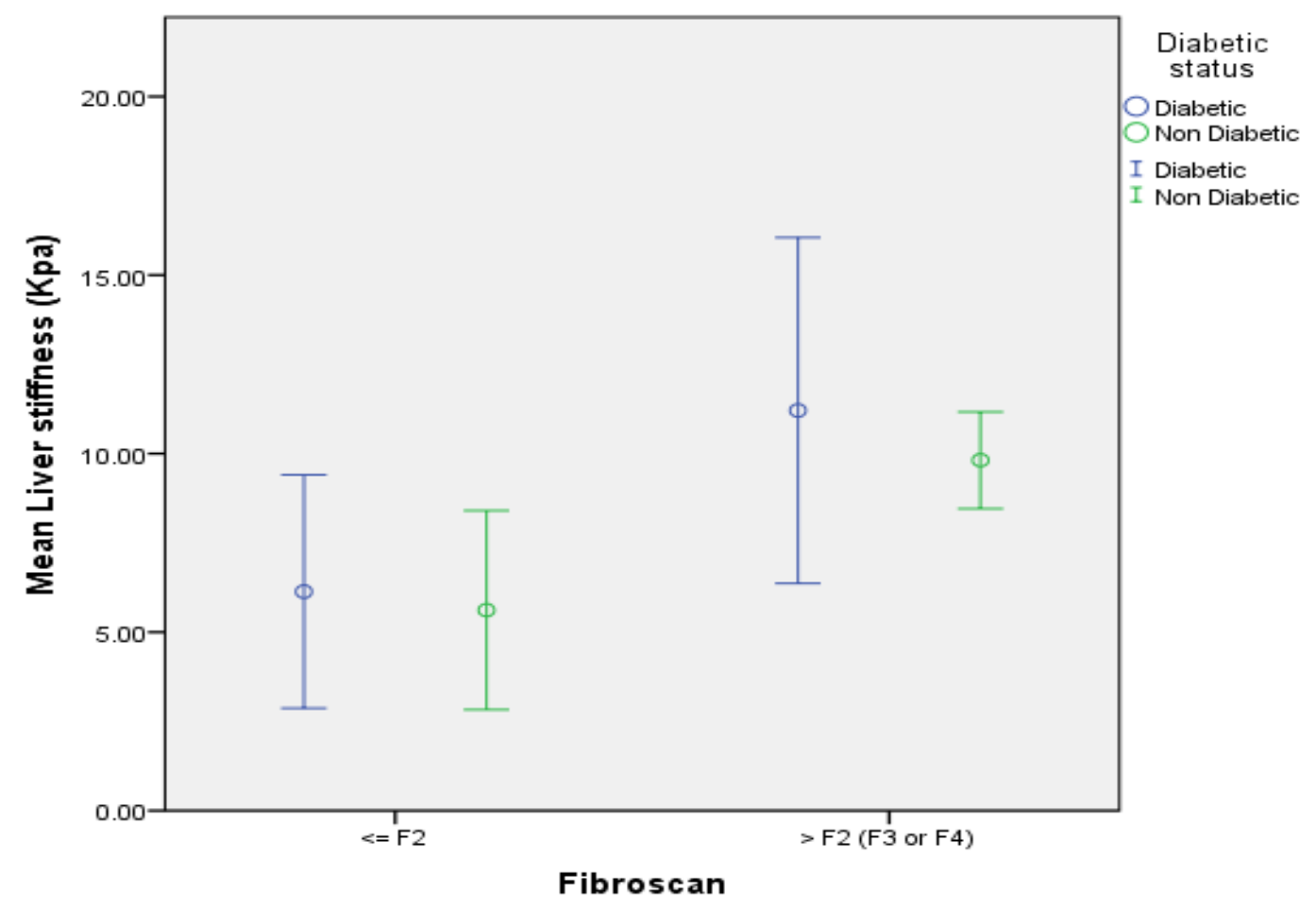

Error Bars: +/- 2 SD

Figure (2): Difference between Fib-4 score and liver fibrosis stages

There was significant positive correlation between liver stiffness score and all patients with (NAFLD and Fib-4) score (Table 5).

Table (5): Correlation between liver stiffness and NAFLD fibrosis score among diabetics and non-diabetics

\begin{tabular}{|l|l|c|}
\hline & & Liver stiffness values \\
\hline \multirow{2}{*}{$\begin{array}{l}\text { NAFLD All } \\
\text { patients }\end{array}$} & $\mathrm{R}$ & 0.943 \\
\cline { 2 - 3 } Fib 4 All patients & $\mathrm{P}$ value & $<0.001 *$ \\
\cline { 2 - 3 } & $\mathrm{R}$ & 0.968 \\
\cline { 2 - 3 } & $\mathrm{P}$ value & $<0.001 *$ \\
\hline
\end{tabular}

\section{DISCUSSION}

The prevalence of NAFLD was $20-30 \%$ in Europe and in the Middle East, 15\% in the Far East and $10-35 \%$ in the most of the United States, with prevalence population $57-95 \%$ obese, $60-73 \%$ diabetic, up to $50 \%$ hyperlipidemia ${ }^{(\mathbf{1 5})}$.

NAFLD itself is a risk factor for increased morbidity and mortality plus cardiovascular disease and malignancy. Despite all of these, most patients have good prognosis if diagnosed at early stages ${ }^{(\mathbf{1 6})}$.

We have mentioned in the results that there was significant difference between the two groups as regard (Fib-4 and NAFLD fibrosis score), where Fib4 and NAFLD fibrosis score were higher in group I (diabetic) than in group II (non-diabetic) with nonalcoholic fatty liver disease. Previous study by Goh $\boldsymbol{e t}$ al. (17), had reported that the clinical utility of the NAFLD fibrosis score differed between NAFLD patients with and without diabetes, with sensitivity to exclude advanced fibrosis being 90\% of NAFLD patients with DM. Diabetes is associated with inflammation, oxidative stress and excessive production of hepatotoxic cytokines that all are involved in the pathophysiology of non-alcoholic fatty liver disease. Diabetic patients with non-alcoholic fatty liver disease would experience a higher probability of advanced fibrosis and cirrhosis, so their complications and mortality will raise ${ }^{(\mathbf{1 8})}$.

Moreover, liver stiffness was found to be higher in group I (diabetic) than in group II (non-diabetic) with non-alcoholic fatty liver disease and this agrees with a study conducted by Hajiani et al. ${ }^{\left({ }^{(9)}\right)}$, who found that, evaluation of 70 patients with non-alcoholic fatty liver disease cleared that liver stiffness score among diabetic patients was highly significant than nondiabetic patients with non-alcoholic fatty liver disease.

So, it is of importance to detect and assess fatty liver among diabetics in early stages, the detection of early stages of fibrosis can be potentially preventive of bad prognosis by more frequent monitoring and more strict therapeutic interventions.

A significant positive correlation between liver stiffness score and (BMI, Age, F.B.G, 2h postprandial blood glucose and TG) was observed, while there was significant negative correlation between liver stiffness score and (ALT and AST). Previous study reported that body mass index, waist circumference, fasting blood sugar levels, and liver enzymes, had significant positive correlation with liver stiffness whereas triglyceride levels, high-density lipoprotein levels, and duration of diabetes mellitus did not correlate with liver stiffness ${ }^{(\mathbf{2 0})}$. 
The higher mean liver stiffness values with T2DM in our study can be explained in the same context and the metabolic significance of the liver stiffness values were also demonstrated by its positive significant correlation with (BMI, Age, F.B.G, $2 \mathrm{~h}$ postprandial blood glucose and TG). Therefore, liver stiffness values may be an additional parameter that can supplement the traditional variable representing metabolic risk. In addition, NAFLD is now accepted as hepatic manifestation of metabolic syndrome, also the liver stiffness values would likely be used as a unique metabolic parameter that specifically targets the liver.

There was statistically significant difference between Fib-4, liver stiffness score and NAFLD fibrosis score as regard patients with advanced fibrosis (> F2) than those with mild to moderate fibrosis (F2 or less), where Fib-4, liver stiffness score and NAFLD fibrosis score were higher in those with $(>F 2)$ than those with (F2 or less). Previous study by Fallatah $\boldsymbol{e t}$ al. ${ }^{(21)}$, reported that there was a significant difference in the results of liver stiffness scores, APRI and the FIB-4 between patients with advanced fibrosis of more than F2 and those with mild to moderate fibrosis of F2 or under.

There was a significant positive correlation between liver stiffness score and all patients with (NAFLD and Fib-4) score. Previous study reported that there was strong positive correlation between the Fibroscan results and AST/ALT ratio, APRI scores, and FIB-4 scores ${ }^{(\mathbf{2 1})}$.

\section{CONCLUSION}

A combination of Fibroscan, Fib-4 and NAFLD fibrosis score provides a valuable non-invasive method in diagnosis of NAFLD patients, and this can eliminate the need for liver biopsy in patients without clear indication, especially diabetic patients that can help in early diagnosis of NAFLD before development of fibrosis.

Limitations of the study: sample size might be small in view of the NAFLD prevalence data, but this could be compensated by the strict inclusion and exclusion criteria. Another limitation is that liver biopsy, the gold standard for NAFLD diagnosis, was not used in this study, because of complications such a procedure can cause, it should not be recommended for every patients with NAFLD.

\section{REFERENCES}

1. Wong V, Wong G, Choi P et al. (2010): Disease progression of non-alcoholic fatty liver disease: a prospective study with paired liver biopsies at 3 years. Gut, 59: 969-974.

2. Matteoni C, Younossi Z, Gramlich T et al. (1999): Alcoholic fatty liver disease: a spectrum of clinical and pathological severity. Gastroenterology, 116:1413-19.

3. McCullough A (2002): Update on nonalcoholic fatty liver disease. J Clin Gastroenterol., 34: 255-262.
4. Adams L, Lymp J, Sauver J et al. (2005): The natural history of nonalcoholic fatty liver disease: a populationbased cohort study. Gastroenterology, 129: 113-121.

5. Motola D, Caravan $P$, Chung $R$ et al. (2014): Noninvasive biomarkers of liver fibrosis: clinical applications and future directions. Curr Pathobiol Rep., 2(4): 245-256.

6. Trifan A, Stanciu C (2012): Checkmate to liver biopsy in chronic hepatitis C? World J Gastroenterol., 18:5514-20.

7. Smith J, Sterling $\mathbf{R}$ (2009): Systematic review: noninvasive methods of fibrosis analysis in chronic hepatitis $\mathrm{C}$. Aliment Pharmacol Ther., 30: 557-76.

8. Angulo P, Hui J, Marchesini G et al. (2007): The NAFLD fibrosis score: a noninvasive system that identifies liver fibrosis in patients with NAFLD. Hepatology, 45(4):846-54.

9. Sterling R, Lissen E, Clumeck $\mathrm{N}$ et al. (2006): Development of a simple noninvasive index to predict significant fibrosis in patients with $\mathrm{HIV} / \mathrm{HCV}$ coinfection. Hepatology, 43:1317-25.

10. Khov N, Sharma A, Riley T (2014): Bedside ultrasound in the diagnosis of nonalcoholic fatty liver disease. World Journal of Gastroenterology, 20(22): 6821-6825.

11. Friedrich-Rust M, Ong M, Herrmann E et al. (2007): Real-time elastography for noninvasive assessment of liver fibrosis in chronic viral hepatitis. AJR Am J Roentgenol., 188(3):758-64.

12. Sporea I, Sirli R, Deleanu A et al. (2009): S2084 transient elastography (fibroscanr) as compared to real-time elastography (Siemens) in patients with chronic hepatopathies. Gastroenterology, 136(5):327-32.

13. Foucher J, Chanteloup E, Vergniol J et al. (2006): Diagnosis of cirrhosis by transient elastography (Fibroscan): a prospective study. Gut, 55: 403-8.

14. Castera L, Verginol J, Foucher J et al. (2005): prospective comparison of transient elastography, fibrotest, APRI, and liver biopsy for assessment of fibrosis in chronic hepatits C. Gastroenterology, 128: 343-350.

15. Chalasani N, Younossi Z, Lavine J et al. (2012): The diagnosis and management of non-alcoholic fatty liver disease: practice guideline by the American Gastroenterological Association, American Association for the Study of Liver Diseases, and American College of Gastroenterology. Gastroenterology, 142: 1592-1609.

16. Gastaldelli A, Harrison S, Belfort-Aguilar R et al. (2009): Importance of changes in adipose tissue insulin resistance to histological response during thiazolidinedione treatment of patients with nonalcoholic steatohepatitis. Hepatology, 50(4):1087-93.

17. Goh G, Paqadala M, Dasarathy J et al. (2014): Clinical spectrum of non-alcoholic fatty liver disease in diabetic and non-diabetic patients. BBA Clin., 3:141-5.

18. Obika M, Noguchi H (2012): Diagnosis and Evaluation of non-alcoholic fatty liver disease. Exp Diabetes Res., 2012: 145754.

19. Hajiani E, Hashemi S, Masjedizadeh A et al. (2014): Comparison of liver biopsy with Transient Elastography as a non-invasive method for assessment of liver fibrosis. Journal of GHR., 3(3): 1013-1016.

20. Kumar A (2019): Assessment of liver involvement in type 2 diabetes mellitus using fibroscan and correlation with risk factors. In. J. of Advance in Medicine, 6: 3-6.

21. Fallatah H, Akbar H, Fallatah A (2016): Fibroscan Compared to FIB-4, APRI, and AST/ALT Ratio for Assessment of Liver Fibrosis in Saudi Patients With Nonalcoholic Fatty Liver Disease. Hepat Mon., 16(7): e38346. 